\title{
Ramseyan ultrafilters
}

by

\section{Lorenz Halbeisen (Belfast)}

\begin{abstract}
We investigate families of partitions of $\omega$ which are related to special coideals, so-called happy families, and give a dual form of Ramsey ultrafilters in terms of partitions. The combinatorial properties of these partition-ultrafilters, which we call Ramseyan ultrafilters, are similar to those of Ramsey ultrafilters. For example it will be shown that dual Mathias forcing restricted to a Ramseyan ultrafilter has the same features as Mathias forcing restricted to a Ramsey ultrafilter. Further we introduce an ordering on the set of partition-filters and consider the dual form of some cardinal characteristics of the continuum.
\end{abstract}

0. Introduction. The Stone-Čech compactification $\beta \mathbb{N}$ of the natural numbers, or equivalently, the ultrafilters over $\omega$, is a well-studied space (cf. e.g. [vM90] and [CN74]) which has a lot of interesting topological and combinatorial features (cf. [HS98] and [To97]). In the late 1960's, a partial ordering on the non-principal ultrafilters $\beta \mathbb{N} \backslash \mathbb{N}$, the so-called Rudin-Keisler ordering, was established and "small" points with respect to this ordering were investigated rigorously (cf. [Bo70], [B173], [Bl81 1 ] and [La89]). The minimal points have a nice combinatorial characterization which is related to Ramsey's Theorem (cf. [Ra29, Theorem A]) and therefore the ultrafilters which are minimal with respect to the Rudin-Keisler ordering are also called Ramsey ultrafilters (for further characterizations of Ramsey ultrafilters see [BJ95, Chapter 4.5]). Ramsey ultrafilters have combinatorial properties similar to certain families, not necessarily filters, called happy families (cf. [Ma77]), which are very important in the investigation of Mathias forcing (cf. [Ma77]).

2000 Mathematics Subject Classification: Primary 05D05, 05D10; Secondary 03E05, 03E40, 03E17, 03E35.

Key words and phrases: dual Ramsey theory, partitions, filters, happy families, Mathias forcing.

I would like to thank the Swiss National Science Foundation for its support during the period in which the research for this paper has been done. 
From the category-theoretical point of view, subsets of $\omega$ and partitions of $\omega$ are dual to each other (see e.g. [HL $\infty_{1}$, Introduction]), and therefore, it is natural to look for the dualization of statements about subsets of $\omega$ in terms of partitions of $\omega$. In this dualization process, a lot of work is already done. We refer to: $\left[\mathrm{HL}_{1}\right]$ for a dualization of $\beta \mathbb{N}$; [CS84], [Ha98 $\left.{ }_{1}\right]$ and $\left[\mathrm{HL} \infty_{2}\right]$ for the dualization of the Ramsey property and of Mathias forcing; [CS84] for a dualization of Ramsey's Theorem; [CW00] and [Ha98 2 for the dualization of some cardinal characteristics of the continuum.

To investigate partition-filters, a useful tool is missing: the dualization of Ramsey ultrafilters. The aim of this paper is to fill this gap.

\section{Partition-filters}

1.1. Notations and definitions. Most of our set-theoretic notation is standard and can be found in textbooks like [Je78], [Ku83] or [BJ95]. So, we consider a natural number $n$ as an ordinal, in particular $n=\{k: k<n\}$ and $0=\emptyset$, and consequently, the set of natural numbers is denoted by $\omega$. For a set $S, \mathcal{P}(S)$ denotes the power-set of $S$. The notation concerning partitions is not yet standardized. We will use the notation introduced in [Ha98 $]$.

A partition $X$ of a set $S$ consists of pairwise disjoint, non-empty sets, such that $\bigcup X=S$. The elements of a partition are called blocks. Mostly, we will consider partitions of $\omega$, so, if not specified otherwise, the word "partition" refers to a partition of $\omega$.

Most of the partitions considered are infinite, or in other words, contain infinitely many blocks. However, at some places we also have to consider finite partitions, that is, partitions containing only finitely many blocks. The unique partition containing just one block is denoted by $\{\omega\}$. The set of all partitions is denoted by $(\omega) \leq \omega$ and the set of all partitions containing infinitely many blocks is denoted by $(\omega)^{\omega}$.

Let $X$ and $Y$ be two partitions of a set $S$. We say $X$ is coarser than $Y$, or that $Y$ is finer than $X$ (and write $X \sqsubseteq Y$ ), if each block of $X$ is a union of blocks of $Y$. Let $X \sqcap Y$ denote the finest partition of $S$ which is coarser than $X$ and $Y$.

Further, for $n \in \omega$ and a partition $X \in(\omega) \leq \omega$, let $X \sqcap\{n\}$ be the partition we get by gluing together all blocks of $X$ which contain a member of $n$. If $X$ and $Y$ are two partitions, then we write $X \sqsubseteq^{*} Y$ if there is an $n \in \omega$ such that $X \sqcap\{n\} \sqsubseteq Y$.

A set $\mathscr{F} \subseteq(\omega)^{\leq \omega}$ is a partition-filter if the following holds:

(a) $\{\omega\} \notin \mathscr{F}$.

(b) For any $X, Y \in \mathscr{F}$ we have $X \sqcap Y \in \mathscr{F}$.

(c) If $X \in \mathscr{F}$ and $X \sqsubseteq Y \in(\omega) \leq \omega$, then $Y \in \mathscr{F}$. 
A partition-filter $\mathscr{F} \subseteq(\omega) \leq \omega$ is called principal if there is a partition $X \in(\omega) \leq \omega$ such that $\mathscr{F}=\{Y: X \sqsubseteq Y\}$.

A set $\mathscr{U} \subseteq(\omega) \leq \omega$ is a partition-ultrafilter if $\mathscr{U}$ is a partition-filter which is not properly contained in any partition-filter.

Notice that a partition-ultrafilter $\mathscr{U}$ which does not contain a finite partition is always non-principal, and vice versa, a principal partition-ultrafilter always contains a finite partition, in fact it contains a 2-block partition (see $\left[\mathrm{HL} \infty_{1}\right.$, Fact 3.1]). Thus, if $\mathscr{U}$ is a non-principal partition-ultrafilter, $X \in \mathscr{U}$ and $X \sqsubseteq{ }^{*} Y$, then $Y \in \mathscr{U}$.

In what follows we are mostly interested in partition-filters which do not contain a finite partition, or in other words, in partition-filters $\mathscr{F} \subseteq(\omega)^{\omega}$.

For the sake of convenience, we defined the notion of partition-filter only for partition-filters over $\omega$, but it is obvious how to generalize this notion to partition-filters over arbitrary sets $S$ (see also $\left[\mathrm{HL} \infty_{1}\right]$ ).

1.2. An ordering on the set of partition-filters. Let $\mathrm{PF}((\omega) \leq \omega)$ denote the set of all partition-filters. We define a partial ordering on $\operatorname{PF}((\omega) \leq \omega)$ which has some similarities with the Rudin-Keisler ordering on $\beta \mathbb{N} \backslash \mathbb{N}$.

To keep the notation short, for $\mathscr{H} \subseteq \mathcal{P}(\mathcal{P}(\omega))$ and a function $f: \omega \rightarrow \omega$ we define

$$
f^{-1}(\mathscr{H}):=\left\{f^{-1}(X): X \in \mathscr{H}\right\}
$$

where for $X \in \mathscr{H}$ we define

$$
f^{-1}(X):=\left\{f^{-1}(b): b \in X\right\}
$$

with $f^{-1}(b):=\{n: f(n) \in b\}$ for $b \subseteq \omega$.

Let $f: \omega \rightarrow \omega$ be any surjection from $\omega$ onto $\omega$ and let $X \in(\omega) \leq \omega$ be any partition. Then $f(X)$ denotes the finest partition such that whenever $n$ and $m$ lie in the same block of $X$, then $f(n)$ and $f(m)$ lie in the same block of $f(X)$.

For any partition-filter $\mathscr{F} \in \operatorname{PF}((\omega) \leq \omega)$ define

$$
f(\mathscr{F}):=\{Y \in(\omega) \leq \omega: \exists X \in \mathscr{F}(f(X) \sqsubseteq Y)\} .
$$

We define the ordering " $\lesssim$ " on $\operatorname{PF}((\omega) \leq \omega)$ as follows:

$\mathscr{F} \lesssim \mathscr{G}$ if and only if $\mathscr{F}=f(\mathscr{G})$ for some surjection $f: \omega \rightarrow \omega$.

Since the identity map is a surjection and the composition of two surjections is again a surjection, the partial ordering " $\lesssim$ " is reflexive and transitive.

FACT 1.2.1. Let $\mathscr{F}, \mathscr{G} \in \mathrm{PF}\left((\omega)^{\leq \omega}\right)$ and assume $f(\mathscr{G})=\mathscr{F}$ for some surjection $f: \omega \rightarrow \omega$. Then $\mathscr{G} \subseteq f^{-1}(\mathscr{F})$ and $f^{-1}(\mathscr{F}) \in \operatorname{PF}((\omega) \leq \omega)$.

Proof. Let $\mathscr{H}=f^{-1}(\mathscr{F})$, where $f: \omega \rightarrow \omega$ is such that $f(\mathscr{G})=\mathscr{F}$. Since $\mathscr{F}$ is a partition-filter and $f$ is a function, for any $X_{1}, X_{2} \in \mathscr{F}$ we have $X_{1} \sqcap X_{2} \in \mathscr{F}$ and $f^{-1}\left(X_{1} \sqcap X_{2}\right)=f^{-1}\left(X_{1}\right) \sqcap f^{-1}\left(X_{2}\right)$, and therefore, 
$\mathscr{H}$ is a partition-filter. Further, for any $Y \in \mathscr{G}$ we get $f(Y) \in \mathscr{F}$ and $f^{-1}(f(Y)) \sqsubseteq Y$, which implies $\mathscr{G} \subseteq \mathscr{H}$.

The ordering " $\lesssim$ " induces in a natural way an equivalence relation " $\simeq$ " on the set $\mathrm{PF}((\omega) \leq \omega)$ of partition-filters:

$$
\mathscr{F} \simeq \mathscr{G} \text { if and only if } \mathscr{F} \lesssim \mathscr{G} \text { and } \mathscr{G} \lesssim \mathscr{F} .
$$

So, the ordering " $\lesssim$ " induces a partial ordering on the set of equivalence classes of partition-filters. Concerning partition-ultrafilters, we get the following.

FACT 1.2.2. Let $\mathscr{U}, \mathscr{V} \in \operatorname{PUF}\left((\omega)^{\leq \omega}\right)$ and assume that $\mathscr{U}$ is principal or contains a partition all of whose blocks are infinite. If $\mathscr{U} \simeq \mathscr{V}$, then there is a permutation $h$ of $\omega$ such that $h(\mathscr{U})=\mathscr{V}$.

Proof. Because $\mathscr{U} \lesssim \mathscr{V}$ and $\mathscr{V} \lesssim \mathscr{U}$, there are surjections $f$ and $g$ from $\omega$ onto $\omega$ such that $\mathscr{V}=f(\mathscr{U})$ and $\mathscr{U}=g(\mathscr{V})$, and because $\mathscr{U}$ and $\mathscr{V}$ are both partition-ultrafilters, by Fact 1.2 .1 we get $\mathscr{U}=f^{-1}(\mathscr{V})$ and $\mathscr{V}=g^{-1}(\mathscr{U})$.

First assume that $\mathscr{U}$ is principal and therefore contains a 2-block partition $X=\left\{b_{0}, b_{1}\right\}$. Because $g^{-1}(X) \in \mathscr{V}$, the partition-ultrafilter $\mathscr{V}$ is also principal and we get $\mathscr{V}=\left\{Y \in(\omega) \leq \omega: g^{-1}(X) \sqsubseteq Y\right\}$, where $g^{-1}(X)=$ $\left\{g^{-1}\left(b_{0}\right), g^{-1}\left(b_{1}\right)\right\}=:\left\{c_{0}, c_{1}\right\}$. Now, because $\mathscr{U}=f^{-1}(\mathscr{V})$, we must have $f^{-1}\left(g^{-1}(X)\right)=X$, which implies $f^{-1}\left(g^{-1}\left(b_{i}\right)\right) \in\left\{b_{0}, b_{1}\right\}$ (for $\left.i \in\{0,1\}\right)$. If one of the blocks of $X$ is finite, say $b_{0}$, then $\left.f\right|_{b_{0}}$ as well as $\left.g\right|_{f\left(b_{0}\right)}$ must be one-to-one, and therefore, $b_{0}$ has the same cardinality as $c_{0}$. Hence, whether or not any of the blocks of $X$ is finite, we can define a permutation $h$ of $\omega$ such that $h\left(b_{0}\right)=c_{0}$ and $h\left(b_{1}\right)=c_{1}$, which implies $h(\mathscr{U})=\mathscr{V}$.

Now assume that $\mathscr{U}$ contains a partition $X=\left\{b_{i}: i \in \omega\right\}$ all of whose blocks $b_{i}$ are infinite. Because $g$ is a surjection, $g^{-1}(X)$, which is a member of $\mathscr{V}$, is a partition all of whose blocks are infinite. Let $h$ be a permutation of $\omega$ such that $h\left(b_{i}\right)=g^{-1}\left(b_{i}\right)$. Take any $Y \in \mathscr{V}$ with $Y \sqsubseteq g^{-1}(X)$. By the definition of $h$ we have $h^{-1}(Y)=g(Y)$ and since $\mathscr{U}=g(\mathscr{V})$ there is a $Z \in \mathscr{U}$ such that $g(Y)=Z$, which implies $h(Z)=Y$, hence, $h(\mathscr{U})=\mathscr{V} . \dashv$

The following proposition shows that " $\lesssim$ " is upward directed (for a similar result concerning the Rudin-Keisler ordering see [Bl73, p. 147]).

FACT 1.2.3. For any partition-filters $\mathscr{D}, \mathscr{E} \in \mathrm{PF}((\omega) \leq \omega)$, there is a partition-filter $\mathscr{F} \in \mathrm{PF}((\omega) \leq \omega)$ such that $\mathscr{D} \lesssim \mathscr{F}$ and $\mathscr{E} \lesssim \mathscr{F}$.

Proof. Let $\varrho_{1}$ and $\varrho_{2}$ be functions from $\omega$ into $\omega$ defined by $\varrho_{1}(n):=2 n$ and $\varrho_{2}(n):=2 n+1$. For a partition $X$ and $i \in\{0,1\}$, let $\varrho_{i}(X):=\left\{\varrho_{i}(b)\right.$ : $b \in X\}$, where $\varrho_{i}(b):=\left\{\varrho_{i}(n): n \in b\right\}$. Now, take any two partition-filters $\mathscr{D}, \mathscr{E} \in \mathrm{PF}\left((\omega)^{\leq \omega}\right)$ and define

$$
\mathscr{F}:=\left\{\varrho_{1}(X) \cup \varrho_{2}(Y): X \in \mathscr{D} \wedge Y \in \mathscr{E}\right\} .
$$


Clearly, this defines a partition-filter. Define two surjections $f$ and $g$ from $\omega$ onto $\omega$ as follows:

$$
f(n)=\left\{\begin{array}{ll}
n / 2 & \text { if } n \text { is even, } \\
0 & \text { otherwise. }
\end{array} \quad g(n)= \begin{cases}(n-1) / 2 & \text { if } n \text { is odd } \\
0 & \text { otherwise }\end{cases}\right.
$$

It is easy to verify that $f(\mathscr{F})=\mathscr{D}$ and $g(\mathscr{F})=\mathscr{E}$, which implies $\mathscr{D} \lesssim \mathscr{F}$ and $\mathscr{E} \lesssim \mathscr{F}$.

\section{Ramseyan ultrafilters}

2.1. Coloring segments. If $X$ is a partition of a set $S$, then we say that $S$ is the domain of $X$, written $\operatorname{dom}(X)=S$. The set of all partitions of natural numbers $n \in \omega$, called segments, is denoted by $(\mathbb{N})$. Thus, $s \in(\mathbb{N})$ implies $\operatorname{dom}(s) \in \omega$. In particular, $\emptyset$ is the unique partition of 0 and $\{\{\emptyset\}\}=\{1\}$ is the unique partition of 1 . For $s \in(\mathbb{N}),|s|$ denotes the cardinality of $s$, which simply means the number of blocks of $s$, and $\bigcup s:=\{\operatorname{dom}(s)\}$.

For a set $b \subseteq \omega$, let $\min (b)$ be the least element of $b$, and for a set $P \subseteq \mathcal{P}(\omega)$, let $\operatorname{Min}(P):=\{\min (b): b \in P\}$. Further, for a finite set $b \subseteq \omega$, let $\max (b)$ be the greatest element of $b$. For $X \in(\omega) \leq \omega, s \in(\mathbb{N})$ and $n \in \omega$, let $X(n)$ and $s(n)$ be the $n$th block of $X$ and $s$, respectively, where we start counting with 0 and assume that the blocks are ordered by their least element.

Let $s, t \in(\mathbb{N})$ and $X \in(\omega) \leq \omega$. We write $s \sqsubseteq X$ if each block $b \in s$ is the union of some sets $b_{i} \cap \operatorname{dom}(s)$, where each $b_{i}$ is a block of $X$; we write $s \preccurlyeq t$ and $s \preccurlyeq X$ if for each $b \in s$ there is a $c_{b} \in t$ and a $d_{b} \in X$, respectively, such that $b=c_{b} \cap \operatorname{dom}(s)=d_{b} \cap \operatorname{dom}(s)$ (notice that $s \preccurlyeq t$ implies $\operatorname{dom}(s) \subseteq \operatorname{dom}(t))$; and for $s \sqsubseteq X, s \sqcap X$ denotes the finest partition $Y \in(\omega) \leq \omega$ such that $s \preccurlyeq Y \sqsubseteq X$.

For $s \in(\mathbb{N})$, let $s^{*}$ denote the partition $s \cup\{\{\operatorname{dom}(s)\}\}$. In particular, $\emptyset^{*}=\{1\}$. Notice that $\left|s^{*}\right|=|s|+1$.

For $s \in(\mathbb{N})$ and $X \in(\omega)^{\omega}$ with $s \sqsubseteq X$, let

$$
(s, X)^{\omega}:=\left\{Y \in(\omega)^{\omega}: s \preccurlyeq Y \sqsubseteq X\right\} .
$$

A set $(s, X)^{\omega}$, where $s$ and $X$ are as above, is called a dual Ellentuck neighborhood (cf. [CS84, p. 275]). In particular, $(\emptyset, X)^{\omega}=(\{1\}, X)^{\omega}=$ : $(X)^{\omega}$.

For $n \in \omega,(\omega)^{n *}$ denotes the set of all $u \in(\mathbb{N})$ such that $|u|=n$. Further, for $n \in \omega$ and $X \in(\omega)^{\omega}$ let

$$
(X)^{n *}:=\left\{u \in(\mathbb{N}):|u|=n \wedge u^{*} \sqsubseteq X\right\} ;
$$

and if $s \in(\mathbb{N})$ is such that $|s| \leq n$ and $s \sqsubseteq X$, let

$$
(s, X)^{n *}:=\left\{u \in(\mathbb{N}):|u|=n \wedge s \preccurlyeq u \wedge u^{*} \sqsubseteq X\right\} .
$$

From the so-called Dual Ramsey Theorem of Carlson and Simpson (Theorem 1.2 of [CS84]), we get the following. 
Proposition 2.1.1. For any coloring of $(\omega)^{(n+1) *}$ with $r+1$ colors, where $r, n \in \omega$, and for any $Z \in(\omega)^{\omega}$, there is an infinite partition $X \in(Z)^{\omega}$ such that $(X)^{(n+1) *}$ is monochromatic.

This combinatorial result is the dualization of Ramsey's Theorem [Ra29, Theorem A] in terms of partitions.

We say that a surjection $f: \omega \rightarrow \omega$ respects the partition $X \in(\omega)^{\omega}$ if $f^{-1}(f(X))=X$; otherwise, we say that it disregards $X$. If $f^{-1}(f(X))=$ $\{\omega\}$, then we say that $f$ completely disregards $X$.

Lemma 2.1.2. For any surjection $f: \omega \rightarrow \omega$ and for any $Z \in(\omega)^{\omega}$, there is a partition $X \in(Z)^{\omega}$ such that $f$ either respects or completely disregards $X$.

Proof. For a surjection $f: \omega \rightarrow \omega$, define the coloring $\pi:(\omega)^{2 *} \rightarrow\{0,1\}$ as follows. $\pi(s):=0$ if and only if $f(s(0)) \cap f(s(1))=\emptyset$. By Proposition 2.1.1, there is a partition $X \in(Z)^{\omega}$ such that $(X)^{2 *}$ is monochromatic with respect to $\pi$, which implies that $f$ respects $X$ if $\left.\pi\right|_{(X)^{2 *}}=\{0\}$, and completely disregards $X$ if $\left.\pi\right|_{(X)^{2 *}}=\{1\}$.

In what follows we will use a slightly stronger version of Proposition 2.1.1, which is given in the following two corollaries.

COROLlary 2.1.3. For any coloring of $(\omega)^{(n+k+1) *}$ with $r+1$ colors, where $r, n, k \in \omega$, and for any dual Ellentuck neighborhood $(s, Y)^{\omega}$, where $|s|=n+1$, there is an infinite partition $X \in(s, Y)^{\omega}$ such that $(s, X)^{(n+k+1) *}$ is monochromatic.

Proof. Let $(s, Y)^{\omega}$ be any dual Ellentuck neighborhood with $|s|=n+1$ $\geq 1$. Set $Y^{\prime}:=s \sqcap Y, R:=\bigcup_{i<n+1} Y^{\prime}(i)$ and $Y_{R}:=Y^{\prime} \backslash\left\{Y^{\prime}(i): i<n+1\right\}$, and take any order-preserving bijection $f: \omega \backslash R \rightarrow \omega$. Then $Z:=f\left(Y_{R}\right)$ is an infinite partition of $\omega$. For $u \in(Z)^{(n+k+1) *}$ we define $\xi(u) \in(s, Y)^{(n+k+1) *}$ as follows: $\operatorname{dom}(\xi(u)):=f^{-1}(\operatorname{dom}(u))$ and for $i<n+k+1$,

$$
\xi(u)(i):= \begin{cases}\left(Y^{\prime}(i) \cap \operatorname{dom}(u)\right) \cup f^{-1}(u(i)) & \text { for } i<n+1, \\ f^{-1}(u(i)) & \text { otherwise. }\end{cases}
$$

Let $\pi:(\omega)^{(n+k+1) *} \rightarrow r+1$ be any coloring. Define $\tau:(\omega)^{(n+k+1) *} \rightarrow r+1$ by stipulating $\tau(u):=\pi(\xi(u))$. By Proposition 2.1.1 there is an infinite partition $X^{\prime} \in(Z)^{\omega}$ such that $\left(X^{\prime}\right)^{(n+k+1) *}$ is monochromatic with respect to the coloring $\tau$. Now let $X \in(\omega)^{\omega}$ be such that

$$
X(i):= \begin{cases}Y^{\prime}(i) \cup f^{-1}\left(X^{\prime}(i)\right) & \text { for } i<n+1, \\ f^{-1}\left(X^{\prime}(i)\right) & \text { otherwise. }\end{cases}
$$

Then, by definition of $\tau$ and $X^{\prime}, X \in(s, Y)^{\omega}$ and $(s, X)^{(n+k+1) *}$ is monochromatic with respect to $\pi$. 
Corollary 2.1.4. For any coloring of $\bigcup_{n \in \omega}(\omega)^{(n+k+1) *}$ with $r+1$ colors, where $r, k \in \omega$, and for any $Z \in(\omega)^{\omega}$, there is an infinite partition $X \in(Z)^{\omega}$ such that for any $n \in \omega$ and for any $s \preccurlyeq X$ with $|s|=n+1$, $(s, X)^{(n+k+1) *}$ is monochromatic.

Proof. Using Corollary 2.1.3 repeatedly, we can construct the partition $X \in(\omega)^{\omega}$ straightforwardly by induction on $n$.

We say that a family $\mathscr{C} \subseteq(\omega)^{\omega}$ has the segment-coloring property if for every coloring of $\bigcup_{n \in \omega}(\omega)^{(n+k+1) *}$ with $r+1$ colors, where $r, k \in \omega$, and for any $Z \in \mathscr{C}$ there is an infinite partition $X \in(Z)^{\omega} \cap \mathscr{C}$ such that for any $n \in \omega$ and for any $s \preccurlyeq X$ with $|s|=n+1,(s, X)^{(n+k+1) *}$ is monochromatic.

If a partition-ultrafilter $\mathscr{U} \in \operatorname{PUF}\left((\omega)^{\omega}\right)$ has the segment-coloring property, then it is called a Ramseyan ultrafilter.

The next lemma shows that every partition-filter $\mathscr{F} \in \mathrm{PF}\left((\omega)^{\omega}\right)$ which has the segment-coloring property is a partition-ultrafilter. We have a similar result for Ramsey filters over $\omega$, since every Ramsey filter is an ultrafilter.

LEMMA 2.1.5. If $\mathscr{F} \subseteq(\omega)^{\omega}$ is a partition-filter which has the segmentcoloring property, then $\mathscr{F} \subseteq(\omega)^{\omega}$ is a partition-ultrafilter.

Proof. Take any $Z \in(\omega)^{\omega}$ such that for any $X \in \mathscr{F}, Z \sqcap X \in(\omega)^{\omega}$. Define the coloring $\pi:(\omega)^{2 *} \rightarrow\{0,1\}$ by stipulating $\pi(u)=0$ if and only if $u \in(Z)^{2 *}$. Because $\mathscr{F}$ has the segment-coloring property, there is a partition $X \in \mathscr{F}$ such that $(X)^{2 *}$ is monochromatic with respect to $\pi$, which implies that $X \sqsubseteq Z$ if $\left.\pi\right|_{(X)^{2 *}}=\{0\}$, and $X \sqcap Z=\{\omega\}$ if $\left.\pi\right|_{(X)^{2 *}}=\{1\}$. By the choice of $Z$ we must have $X \sqsubseteq Z$, thus $Z \in \mathscr{F}$, since $\mathscr{F}$ is a partition-filter. $\dashv$

The following lemma gives a relation between Ramseyan and Ramsey ultrafilters.

LEMMA 2.1.6. If $\mathscr{U}$ is a Ramseyan ultrafilter, then $\{\operatorname{Min}(X) \backslash\{0\}$ : $X \in \mathscr{U}\}$ is a Ramsey ultrafilter over $\omega$ (to be pedantic, one should say "over $\omega \backslash\{0\} ")$.

Proof. Let $\tau:[\omega]^{n} \rightarrow r$ be any coloring of the $n$-element subsets of $\omega$ with $r$ colors, where $n$ and $r$ are positive natural numbers. Define $\pi:(\omega)^{n *} \rightarrow r$ by stipulating $\pi(s):=\tau\left(\operatorname{Min}\left(s^{*}\right) \backslash\{0\}\right)$. Take $X \in \mathscr{U}$ such that $(X)^{n *}$ is monochromatic with respect to $\pi$. Then, by the definition of $\pi$, the set $[\operatorname{Min}(X) \backslash\{0\}]^{n}$ is monochromatic with respect to $\tau$.

Ramsey ultrafilters over $\omega$ are the minimal points of the Rudin-Keisler ordering on $\beta \mathbb{N} \backslash \mathbb{N}$. This fact can also be expressed by saying that a nonprincipal ultrafilter $\mathcal{U}$ is a Ramsey ultrafilter if and only if any function $g: \omega \rightarrow \omega$ is either constant or one-to-one on some set of $\mathcal{U}$. By Lemma 2.1.2, we get a similar result for Ramseyan ultrafilters with respect to the ordering "ふ". 
THEOREM 2.1.7. If $\mathscr{U}$ is a Ramseyan ultrafilter, then for any surjection $f: \omega \rightarrow \omega$ there is an $X \in \mathscr{U}$ such that $f$ either respects or completely disregards $X$.

Proof. The proof is the same as the proof of Lemma 2.1.2, but restricted to the partition-ultrafilter $\mathscr{U}$.

2.2. On the existence of Ramseyan ultrafilters. As we have seen in Lemma 2.1.6, every Ramseyan ultrafilter induces a Ramsey ultrafilter over $\omega$. It is not clear if the converse holds as well. However, Ramseyan ultrafilters are always forceable: Let $\mathbb{U}^{b}$ be the forcing notion consisting of infinite partitions, stipulating $X \leq Y \Leftrightarrow X \sqsubseteq^{*} Y$. Then $\mathbb{U}^{b}$ is the natural dualization of the forcing notion $\left\langle\mathcal{P}(\omega) /\right.$ fin, $\left.\subseteq^{*}\right\rangle$, denoted by $\mathbb{U}$ in what follows, and it is not hard to see that if $\mathscr{G}$ is $\mathbb{U}^{\mathrm{b}}$-generic over $\mathbf{V}$, then $\mathscr{G}$ is a Ramseyan ultrafilter in $\mathbf{V}[\mathscr{G}]$. Since $\mathbb{U}^{b}$ is $\sigma$-closed, we deduce (see below) that Ramseyan ultrafilters exist if we assume the continuum hypothesis (denoted by $\mathrm{CH}$ ). On the other hand we know by Lemma 2.1.6 that Ramseyan ultrafilters cannot exist if there are no Ramsey ultrafilters. Kenneth Kunen proved (cf. [Je78, Theorem 91]) that it is consistent with ZFC that Ramsey ultrafilters do not exist. We wish to mention that Saharon Shelah showed that even $p$-points, which are weaker ultrafilters than Ramsey ultrafilters, may not exist (see [Sh98, VI $\S 4]$ ). He also proved that it is possible that-up to isomorphisms - there exists a unique Ramsey ultrafilter (see [Sh98, VI §5]).

In the following, $\mathfrak{c}$ denotes the cardinality of the continuum and $2^{\mathfrak{c}}$ denotes the cardinality of its power-set.

Andreas Blass proved that Martin's Axiom, denoted by MA, implies the existence of $2^{\mathfrak{c}}$ Ramsey ultrafilters (see [Bl73, Theorem 2]). He mentions in that paper that with $\mathrm{CH}$ in place of $\mathrm{MA}$, this result is due to Keisler, and with 1 in place of $2^{\mathfrak{c}}$, to Booth (cf. [Bo70, Theorem 4.14]). Further he mentions that his proof is essentially the union of Keisler's and Booth's proofs. However, Blass' proof uses at a crucial point the fact that MA implies that the tower number is equal to $c$. Such a result does not hold for partitions, because Timothy Carlson proved that the dual-tower number is equal to $\aleph_{1}$ (see [Mt86, Proposition 4.3]). So, concerning the existence of Ramseyan ultrafilters under MA, we cannot simply translate the proof of Blass, and it seems that MA and sets of partitions are quite unrelated. But as mentioned above, if one assumes $\mathrm{CH}$, then Ramseyan ultrafilters exist. Moreover, for the equivalence relation " $\simeq$ " (defined in Section 1.2) we get the following (for a similar result for the Rudin-Keisler ordering see [B173, p. 149]).

TheOREM 2.2.1. $\mathrm{CH}$ implies the existence of $2^{\mathrm{c}}$ pairwise non-equivalent Ramseyan ultrafilters. 
Proof. Assume $\mathbf{V} \models \mathrm{CH}$. Let $\chi$ be large enough such that $\mathcal{P}\left((\omega)^{\omega}\right) \in$ $H(\chi)$, i.e., the power-set of $(\omega)^{\omega}$ (in $\mathbf{V}$ ) is hereditarily of size $<\chi$. Let $\mathbf{N}$ be an elementary submodel of $\langle H(\chi), \in\rangle$ with $|\mathbf{N}|=\aleph_{1}$, containing all reals (or equivalently, all partitions) of $\mathbf{V}$. We consider the forcing notion $\mathbb{U}^{b}$ in the model $\mathbf{N}$. Since $|\mathbf{N}|=\aleph_{1}$, in $\mathbf{V}$ there is an enumeration $\left\{D_{\alpha} \subseteq(\omega)^{\omega}\right.$ : $\left.\alpha<\omega_{1}\right\}$ of all dense sets of $\mathbb{U}^{b}$ which lie in $\mathbf{N}$. For any $Z \in(\omega)^{\omega} \cap \mathbf{V}$, let $Y_{Z}^{\alpha, 0}, Y_{Z}^{\alpha, 1} \in D_{\alpha}$ be such that $Y_{Z}^{\alpha, 0} \sqsubseteq^{*} Z, Y_{Z}^{\alpha, 1} \sqsubseteq^{*} Z$ and $Y_{Z}^{\alpha, 0} \sqcap Y_{Z}^{\alpha, 1} \notin(\omega)^{\omega}$ (since $D_{\alpha}$ is dense, such partitions exist). For any function $\zeta: \mathfrak{c} \rightarrow\{0,1\}$ we can construct a set $H_{\zeta}=\left\{X_{\alpha}: \alpha<\omega_{1}\right\}$ in $\mathbf{V}$ such that for all $\beta<$ $\alpha<\omega_{1}$ we have $X_{\alpha} \sqsubseteq^{*} Y_{X_{\beta}}^{\beta, \zeta(\beta)}$. By construction, for any function $\zeta$, the set $G_{\zeta}:=\left\{X \in(\omega)^{\omega}: X_{\alpha} \sqsubseteq^{*} X\right.$ for some $\left.X_{\alpha} \in H_{\zeta}\right\}$ is $\mathbb{U}^{b}$-generic over $\mathbf{N}$, thus, a Ramseyan ultrafilter in $\mathbf{N}\left[G_{\zeta}\right]$, and since $\mathbb{U}^{b}$ is $\sigma$-closed and therefore adds no new reals, $G_{\zeta}$ is also a Ramseyan ultrafilter in V. Furthermore, if $\zeta \neq \zeta^{\prime}$, then the Ramseyan ultrafilters $G_{\zeta}$ and $G_{\zeta^{\prime}}$ are different (consider the partitions $X_{\beta+1} \in H_{\zeta}$ and $X_{\beta+1}^{\prime} \in H_{\zeta^{\prime}}$, where $\zeta(\beta) \neq \zeta^{\prime}(\beta)$ ). Hence, in $\mathbf{V}$, there are $2^{\mathfrak{c}}$ Ramseyan ultrafilters. Because there are only $\mathfrak{c}$ surjections from $\omega$ onto $\omega$, no equivalence class (w.r.t. " $\simeq$ ") can contain more than $\mathfrak{c}$ Ramseyan ultrafilters, so, in $\mathbf{V}$, there must be $2^{\mathfrak{c}}$ pairwise non-equivalent Ramseyan ultrafilters.

\section{The happy families' relatives}

3.1. Relatively happy families. As we will see below, the partition-families which have the segment-coloring property are related to special coideals, so-called happy families, which were introduced and rigorously investigated by Adrian Mathias in [Ma77]. So, partition-families with the segmentcoloring property can be considered as "relatives of happy families".

Let us first consider the definition of Mathias' happy families.

Let $[\omega]^{\omega}$ be the set of all infinite subsets of $\omega$, and let $[\omega]^{<\omega}$ be the set of all finite subsets of $\omega$. A set $\mathcal{I} \subseteq \mathcal{P}(\omega)$ is a free ideal if $\mathcal{I}$ is an ideal which contains the Fréchet ideal $[\omega]^{<\omega}$. A set $\mathcal{F} \subseteq \mathcal{P}(\omega)$ is a free filter if $\{y: \omega \backslash y \in \mathcal{F}\}$ is an ideal containing the Fréchet ideal. For $a \in[\omega]^{<\omega}$, let $a^{*}:=\max \{n+1: n \in a\}$, in particular, $0^{*}=0$. For $x, y \in \mathcal{P}(\omega)$ we write $y \subseteq^{*} x$ if $y \backslash x \in[\omega]^{<\omega}$. For a set $\mathcal{B} \subseteq \mathcal{P}(\omega)$, let fil $(\mathcal{B})$ be the free filter generated by $\mathcal{B}$; so $x \in \operatorname{fil}(\mathcal{B})$ if and only if there is a finite set $y_{0}, \ldots, y_{n} \in \mathcal{B}$ such that $y_{0} \cap \ldots \cap y_{n} \subseteq^{*} x$.

A set $x \subseteq \omega$ is said to diagonalize the family $\left\{x_{a}: a \in[\omega]<\omega\right\}$ if $x \subseteq x_{0}$ and for all $a \in[\omega]^{<\omega}$, if $\max (a) \in x$, then $x \backslash a^{*} \subseteq x_{a}$.

The family $\mathcal{A} \subseteq \mathcal{P}(\omega)$ is happy if $\mathcal{P}(\omega) \backslash \mathcal{A}$ is a free ideal and whenever fil $\left\{x_{a}: a \in[\omega]^{<\omega}\right\} \subseteq \mathcal{A}$, there is an $x \in \mathcal{A}$ which diagonalizes $\left\{x_{a}: a \in\right.$ $\left.[\omega]^{<\omega}\right\}$. 
In terms of happy families one can define Ramsey ultrafilters as follows: A Ramsey ultrafilter is an ultrafilter that is also a happy family.

Now we turn back to partitions. The Fréchet ideal corresponds to the set of finite partitions, and therefore, the notion of a free filter corresponds to partition-filters containing only infinite partitions, hence, to partition-filters $\mathscr{F} \subseteq(\omega)^{\omega}$.

For a set $\mathscr{B} \subseteq(\omega)^{\omega}$, let fil $(\mathscr{B})$ be the partition-filter generated by $\mathscr{B}$; so $X \in \operatorname{fil}(\mathscr{B})$ if and only if there is a finite set of partitions $Y_{0}, \ldots, Y_{n} \in \mathscr{B}$ such that $Y_{0} \sqcap \ldots \sqcap Y_{n} \sqsubseteq^{*} X$.

A partition $X$ is said to diagonalize the family $\left\{X_{s}: s \in(\mathbb{N})\right\}$ if $X \sqsubseteq X_{\emptyset}$ and for all $s \in(\mathbb{N})$, if $s^{*} \preccurlyeq X$, then $\bigcup s^{*} \sqcap X \sqsubseteq X_{s}$.

The family $\mathscr{A} \subseteq(\omega)^{\omega}$ is relatively happy if whenever fil $\left\{X_{s}: s \in(\mathbb{N})\right\}$ $\subseteq \mathscr{A}$, there is an $X \in \mathscr{A}$ which diagonalizes $\left\{X_{s}: s \in(\mathbb{N})\right\}$.

An example of a relatively happy family is $(\omega)^{\omega}$, the set of all infinite partitions (cf. [Ma77, Example 0.2]). Another example of a much smaller relatively happy family is given in the following theorem (cf. [Ma77, p. 63]).

THEOREM 3.1.1. Every Ramseyan ultrafilter is relatively happy.

Proof. Let $\mathscr{U} \subseteq(\omega)^{\omega}$ be a partition-ultrafilter which has the segmentcoloring property and let $\left\{X_{s}: s \in(\mathbb{N})\right\} \subseteq \mathscr{U}$ be any family. Since $\mathscr{U}$ is a partition-filter, we obviously have fil $\left\{X_{s}: s \in(\mathbb{N})\right\} \subseteq \mathscr{U}$. For $t \in(\mathbb{N})$ with $|t| \geq 2$, let $s_{t}$ be such that $s_{t}^{*} \preccurlyeq t$ and $\left|s_{t}\right|=|t|-2$. Define the coloring $\pi: \bigcup_{n \in \omega}(\omega)^{(n+2) *} \rightarrow\{0,1\}$ by stipulating

$$
\pi(t):= \begin{cases}0 & \text { if } \bigcup s_{t}^{*} \sqcap t^{*} \sqsubseteq X_{s_{t}} \\ 1 & \text { otherwise. }\end{cases}
$$

Let $X \in\left(X_{\emptyset}\right)^{\omega} \cap \mathscr{U}$ be such that for any $n \in \omega$ and for any $s^{*} \preccurlyeq X$ with $|s|=n,\left(s^{*}, X\right)^{(n+2) *}$ is monochromatic with respect to $\pi$. Take any $s^{*} \preccurlyeq X$. Since $\left(s^{*}, X\right)^{(|s|+2) *}$ is monochromatic with respect to $\pi$, each $t^{*} \sqsubseteq X$ with $s^{*} \preccurlyeq t$ and $|t|=|s|+2$ gets the same color. Hence, for all such $t$ 's we have either $\bigcup s^{*} \sqcap t^{*} \sqsubseteq X_{s}$, which implies $X \sqsubseteq^{*} X_{s}$, or $\bigcup s^{*} \sqcap t^{*} \nsubseteq X_{s}$, which implies $X \sqcap X_{s} \notin(\omega)^{\omega}$. The latter is impossible, since it contradicts the assumption that $\mathscr{U}$ is a partition-filter. So, we are always in the former case, which completes the proof.

3.2. A game characterization. There is a characterization of happy ultrafilters over $\omega$, i.e., of Ramsey ultrafilters, in terms of games (cf. [BJ95, Theorem 4.5.3]). We get a similar characterization for relatively happy partition-ultrafilters. 
Let $\mathscr{U}$ be a partition-ultrafilter. Define a game $G(\mathscr{U})$ played by players I and II as follows:

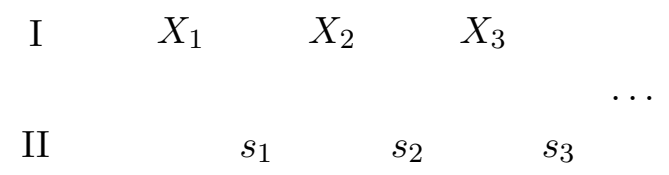

On the $n$th move, player I plays a partition $X_{n} \in \mathscr{U}$. Player II responds with a segment $s_{n} \in(\mathbb{N})$ such that $\left|s_{n}\right|=n, s_{n-1}^{*} \preccurlyeq s_{n}$ and $\bigcup s_{m}^{*} \sqcap s_{n}^{*} \sqsubseteq$ $X_{m+1}$ for all $m<n$, where $s_{0}:=\emptyset$. Player I wins if and only if the unique partition $X$ with $s_{n} \preccurlyeq X$ (for all $n$ ) is not in $\mathscr{U}$.

THEOREM 3.2.1. Let $\mathscr{U} \in \operatorname{PUF}\left((\omega)^{\omega}\right)$. Then player I has a winning strategy in $G(\mathscr{U})$ if and only if $\mathscr{U}$ is not relatively happy.

Proof. Assume first that the partition-ultrafilter $\mathscr{U}$ is relatively happy and that $\left\{X_{s}: s \in(\mathbb{N})\right\}$ is a strategy for player I. This means that player I begins with $X_{\emptyset}$ and if $s_{n}$ is the $n$th move of player II, then player I plays $X_{s_{n}}$. Because $\mathscr{U}$ is relatively happy, there is a partition $X \in \mathscr{U}$ which diagonalizes the family $\left\{X_{s}: s \in(\mathbb{N})\right\}$, in particular, $X \sqsubseteq X_{\emptyset}$. Now, by the definition of $X$ and by the rules of the game $G(\mathscr{U})$, player II can play the segments of $X$. More precisely, on the $n$th move player II plays the segment $s_{n}$ so that $\left|s_{n}\right|=n$ and $s_{n}^{*} \preccurlyeq X$. Since $X \in \mathscr{U}$, the strategy $\left\{X_{s}: s \in(\mathbb{N})\right\}$ was not a winning strategy for player I.

Now assume that the strategy $\sigma=\left\{X_{s}: s \in(\mathbb{N})\right\}$ is not a winning strategy for player I. Consider the game where player I is playing according to the strategy $\sigma$. In this game, player II can play segments $s_{n}$ such that the unique partition $X$ with $s_{n} \preccurlyeq X$ (for all $n$ ) is in $\mathscr{U}$. We have to show that $X$ diagonalizes the family $\left\{X_{s}: s \in(\mathbb{N})\right\}$. For $n \in \omega$, let $s_{n} \in(\mathbb{N})$ be such that $s_{n}^{*} \preccurlyeq X$ and $\left|s_{n}\right|=n$. Fix $m \in \omega$; then, by the rules of the game, for any $n>m$ we have $\bigcup s_{m}^{*} \sqcap s_{n}^{*} \sqsubseteq X_{m+1}$, which implies $\bigcup s_{m}^{*} \sqcap X \sqsubseteq X_{m+1}$. Since player I follows the strategy $\sigma$, we have $X_{m+1}=X_{s_{m}}$, and because $m$ was arbitrary, for all $m \in \omega$ we get $\bigcup s_{m}^{*} \sqcap X \sqsubseteq X_{s_{m}}$. Hence, $X$ diagonalizes the family $\left\{X_{s}: s \in(\mathbb{N})\right\}$.

4. The combinatorics of dual Mathias forcing. First we recall the Ellentuck topology on $[\omega]^{\omega}$. For $x \in[\omega]^{\omega}$ and $a \in[\omega]^{<\omega}$ with $x \cap(\max (a)$ $+1)=a$, let $[a, x]^{\omega}:=\left\{y \in[\omega]^{\omega}: a \subseteq y \subseteq x\right\}$, and let the basic open sets on $[\omega]^{\omega}$ be the sets $[a, x]^{\omega}$. These sets are called Ellentuck neighborhoods. The topology induced by the Ellentuck neighborhoods is called the Ellentuck topology (cf. [El74]).

The Mathias forcing $\mathbb{M}$, introduced in [Ma77], consists of ordered pairs $\langle a, x\rangle$ such that $[a, x]^{\omega}$ is an Ellentuck neighborhood and the ordering on $\mathbb{M}$ is defined by stipulating $\langle a, x\rangle \leq\langle b, y\rangle \Leftrightarrow[a, x]^{\omega} \subseteq[b, y]^{\omega}$. 
Mathias forcing restricted to a non-principal ultrafilter $\mathcal{U}$, denoted by $\mathbb{M}_{\mathcal{U}}$, consists of the ordered pairs $\langle a, x\rangle \in \mathbb{M}$ where in addition we require that $x \in \mathcal{U}$.

Mathias forcing has a lot of nice combinatorial properties (some of them are mentioned below) which also hold for Mathias forcing restricted to a Ramsey ultrafilter (see [Ma77]).

The dual Ellentuck topology on $(\omega)^{\omega}$ is the topology induced by the dual Ellentuck neighborhoods (defined in Section 2.1). Now, the dual Mathias forcing $\mathbb{M}^{b}$, introduced in [CS84], is defined similarly to Mathias forcing $\mathbb{M}$, using the dual Ellentuck topology instead of the Ellentuck topology. So, $\mathbb{M}^{b}$ consists of the ordered pairs $\langle s, X\rangle$ such that $(s, X)^{\omega}$ is a dual Ellentuck neighborhood and the ordering on $\mathbb{M}^{b}$ is defined by stipulating $\langle s, X\rangle \leq\langle t, Y\rangle \Leftrightarrow(s, X)^{\omega} \subseteq(t, Y)^{\omega}$.

Dual Mathias forcing restricted to a partition-ultrafilter $\mathscr{U} \in \operatorname{PUF}\left((\omega)^{\omega}\right)$, denoted by $\mathbb{M}_{\mathscr{U}}^{b}$, consists of the ordered pairs $\langle s, X\rangle \in \mathbb{M}^{b}$ where in addition we require that $X \in \mathscr{U}$ (see e.g. [Ha981] and $\left[\mathrm{HL}_{2}\right]$ ).

Both Mathias forcing and dual Mathias forcing are proper forcings. Moreover, both have (i) a decomposition, (ii) pure decision and (iii) the homogeneity property (see e.g. [Ma77], [CS84] and [Ha981]):

(i) Decomposition: $\mathbb{M} \approx \mathbb{U} * \mathbb{M}_{\check{\mathbf{U}}}$, where $\check{\mathbf{U}}$ is the canonical U-name for the $\mathbb{U}$-generic object ( $\mathbb{U}$ as in Section 2.2).

$\mathbb{M}^{b} \approx \mathbb{U}^{b} * \mathbb{M}_{\check{\mathbf{W}}}^{b}$, where $\check{\mathbf{W}}$ is the canonical $\mathbb{U}^{b}$-name for the $\mathbb{U}^{b}$-generic object ( $\mathbb{U}^{b}$ as in Section 2.2).

(ii) Pure decision: For any $\mathbb{M}$-condition $\langle a, x\rangle$ and any sentence $\Phi$ of the forcing language $\mathbb{M}$, there is an $\mathbb{M}$-condition $\langle a, y\rangle \leq\langle a, x\rangle$ such that either $\langle a, y\rangle \|_{\mathbb{M}} \Phi$ or $\langle a, y\rangle \Vdash_{\mathbb{M}} \neg \Phi$.

For any $\mathbb{M}^{b}$-condition $\langle s, X\rangle$ and any sentence $\Phi$ of the forcing language $\mathbb{M}^{b}$, there is an $\mathbb{M}^{b}$-condition $\langle s, Y\rangle \leq\langle s, X\rangle$ such that either $\langle s, Y\rangle \Vdash_{\mathbb{M}^{b}} \Phi$ or $\langle s, Y\rangle \Vdash_{\mathbb{M}^{b}} \neg \Phi$.

(iii) Homogeneity property: If $x_{G}$ is $\mathbb{M}$-generic over $\mathbf{V}$ and $y \in\left[x_{G}\right]^{\omega}$, then $y$ is also $\mathbb{M}$-generic over $\mathbf{V}$.

If $X_{G}$ is $\mathbb{M}^{b}$-generic over $\mathbf{V}$ and $Y \in\left(X_{G}\right)^{\omega}$, then $Y$ is also $\mathbb{M}^{b}$-generic over $\mathbf{V}$.

In $\left[\mathrm{Ha}_{9} 8_{1}\right]$ it is shown that if $\mathscr{F} \subseteq(\omega)^{\omega}$ is a so-called game-family, then $\mathbb{M}_{\mathscr{F}}^{b}$ has pure decision and the homogeneity property ([Ha98 1 , Thm. $4.3 \& 4.4]$ ). Game-families have the segment-coloring-property and therefore, the so-called game-filters, i.e., game-families which are partition-filters, are Ramseyan ultrafilters. Unlike for Ramseyan ultrafilters, it is not clear if $\mathrm{CH}$ implies the existence of game-filters, so, it seems that game-filters are stronger than Ramseyan ultrafilters. However, in what follows we show that 
if $\mathscr{U} \in \operatorname{PUF}\left((\omega)^{\omega}\right)$ is a Ramseyan ultrafilter, then $\mathbb{M}_{\mathscr{U}}^{b}$ has pure decision and the homogeneity property.

Recently, Stevo Todorčević gave an abstract presentation of Ellentuck's theorem by introducing the notion of a quasi-ordering with approximations which admits a finitization and the notion of a Ramsey space. The Abstract Ellentuck Theorem says that a quasi-ordering with approximations which admits a finitization and satisfies certain axioms is a Ramsey space.

Let $\mathscr{U} \in \operatorname{PUF}\left((\omega)^{\omega}\right)$ be a partition-ultrafilter and let " $\sqsubseteq$ " be the quasiordering on $\mathscr{U}$. For each $n \in \omega$, let $p_{n}: \mathscr{U} \rightarrow(\mathbb{N})$ be such that $p_{n}(X)$ is the unique $s$ with $s^{*} \preccurlyeq X$ and $|s|=n$. Let $p$ be the sequence $\left(p_{n}\right)_{n \in \omega}$. It is easy to verify that the triple $(\mathscr{U}, \sqsubseteq, p)$ is a quasi-ordering with approximations. For $n, m \in \omega$ and $X, Y \in \mathscr{U}$ define: $p_{n}(X) \sqsubseteq_{\text {fin }} p_{m}(Y)$ if and only if $\operatorname{dom}\left(p_{n}(X)\right)=\operatorname{dom}\left(p_{m}(Y)\right)$ and $p_{n}(X) \sqsubseteq p_{m}(Y)$. This definition verifies that $(\mathscr{U}, \sqsubseteq, p)$ admits a finitization. If $(s, X)^{\omega}$ is a dual Ellentuck neighborhood and $X \in \mathscr{U}$, then $(s, X)^{\omega} \cap \mathscr{U}$ is called a $\mathscr{U}$-dual Ellentuck neighborhood. The topology on $\mathscr{U}$, induced by the $\mathscr{U}$-dual Ellentuck neighborhoods, is called the $\mathscr{U}$-dual Ellentuck topology. With respect to the $\mathscr{U}$-dual Ellentuck topology, the topological space $\mathscr{U}$ is a Ramsey space if for any subset $S \subseteq \mathscr{U}$ which has the Baire property with respect to the $\mathscr{U}$-dual Ellentuck topology, and for any $\mathscr{U}$-dual Ellentuck neighborhood $(s, Y)^{\omega} \cap \mathscr{U}$, there is a partition $X \in(s, Y)^{\omega} \cap \mathscr{U}$ such that either $(s, X)^{\omega} \cap \mathscr{U} \subseteq S$ or $(s, X)^{\omega} \cap \mathscr{U} \subseteq \mathscr{U} \backslash S$.

Let $\mathscr{U} \in \operatorname{PUF}\left((\omega)^{\omega}\right)$ be a Ramseyan ultrafilter. Since the triple $(\mathscr{U}, \sqsubseteq, p)$ satisfies certain axioms, by Todorčević's Abstract Ellentuck Theorem, the Ramseyan ultrafilter $\mathscr{U}$ with respect to the $\mathscr{U}$-dual Ellentuck topology is a Ramsey space. Moreover, we get the following two results.

THEOREM 4.1. If $\mathscr{U}$ is a Ramseyan ultrafilter, then $\mathbb{M}_{\mathscr{U}}^{b}$ has pure decision.

Proof. Let $\Phi$ be any sentence of the forcing language $\mathbb{M}_{\mathscr{U}}^{b}$. With respect to $\Phi$ we define

$$
\begin{aligned}
& D_{0}:=\left\{Y \in \mathscr{U}: \text { for some } t \preccurlyeq Y,\langle t, Y\rangle \Vdash_{\mathbb{M}_{\mathscr{U}}^{b}} \neg \Phi\right\}, \\
& D_{1}:=\left\{Y \in \mathscr{U}: \text { for some } t \preccurlyeq Y,\langle t, Y\rangle \vdash_{\mathbb{M}_{\mathscr{U}}^{b}} \Phi\right\} .
\end{aligned}
$$

Clearly $D_{0}$ and $D_{1}$ are both open (w.r.t. the $\mathscr{U}$-dual Ellentuck topology) and $D_{0} \cup D_{1}$ is dense (w.r.t. the partial order in $\mathbb{M}_{\mathscr{U}}^{b}$ ). Because $\mathscr{U}$ is a Ramsey space, for any $\mathscr{U}$-dual Ellentuck neighborhood $(s, Y)^{\omega} \cap \mathscr{U}$ there is an $X \in(s, Y)^{\omega} \cap \mathscr{U}$ such that either $(s, X)^{\omega} \cap \mathscr{U} \subseteq D_{0}$ or $(s, X)^{\omega} \cap \mathscr{U} \cap D_{0}=$ $\emptyset$. In the former case we have $\langle s, X\rangle \Vdash_{\mathbb{M}_{\mathscr{U}}^{b}} \neg \Phi$ and we are done. In the latter case we find $X^{\prime} \in(s, X)^{\omega} \cap \mathscr{U}$ such that $\left(s, X^{\prime}\right)^{\omega} \cap \mathscr{U} \subseteq D_{1}$. (Otherwise we would have $\left(s, X^{\prime}\right)^{\omega} \cap \mathscr{U} \cap\left(D_{0} \cup D_{1}\right)=\emptyset$, which is impossible by the density of $D_{0} \cup D_{1}$.) Hence, $\left\langle s, X^{\prime}\right\rangle \Vdash_{\mathbb{M}_{\mathscr{U}}^{b}} \Phi$. 
TheOREM 4.2. If $\mathscr{U}$ is a Ramseyan ultrafilter, then $\mathbb{M}_{\mathscr{U}}^{b}$ has the homogeneity property.

Proof. For a dense set $D \subseteq \mathbb{M}_{\mathscr{U}}^{b}$, let

$$
\bigcup D:=\left\{X \in(\omega)^{\omega}: X \in(s, Y)^{\omega} \text { for some }\langle s, Y\rangle \in D\right\} .
$$

It is clear that a partition $X_{G}$ is $\mathbb{M}_{\mathscr{U}}^{b}$-generic if and only if $X_{G} \in \bigcup D$ for each dense set $D \subseteq \mathbb{M}_{\mathscr{U}}^{b}$. Let $D \subseteq \mathbb{M}_{\mathscr{U}}^{b}$ be an arbitrary dense set and let $D^{\prime}$ be the set of all $\langle s, Z\rangle \in \mathbb{M}_{\mathscr{U}}^{b}$ such that $(t, Z)^{\omega} \subseteq \bigcup D$ for all $t \sqsubseteq s$ with $\operatorname{dom}(t)=\operatorname{dom}(s)$.

First we show that $D^{\prime}$ is dense in $\mathbb{M}_{\mathscr{U}}^{b}$. For this, take an arbitrary $\langle s, W\rangle \in \mathbb{M}_{\mathscr{U}}^{b}$ and let $\left\{t_{i}: 0 \leq i \leq m\right\}$ be an enumeration of all $t \in(\mathbb{N})$ such that $t \sqsubseteq s$ and $\operatorname{dom}(t)=\operatorname{dom}(s)$. Because $D$ is dense in $\mathbb{M}_{\mathscr{U}}^{b}, \cup D$ is open (w.r.t. the $\mathscr{U}$-dual Ellentuck topology), and since $\mathscr{U}$ is a Ramsey space, for every $t_{i}$ we find a $W^{\prime} \in \mathscr{U}$ such that $t_{i} \sqsubseteq W^{\prime}$ and $\left(t_{i}, W^{\prime}\right)^{\omega} \subseteq \bigcup D$. Moreover, if we define $W_{-1}:=W$, for every $i \leq m$ we can choose a partition $W_{i} \in \mathscr{U}$ such that $W_{i} \sqsubseteq W_{i-1}, s \preccurlyeq W_{i}$ and $\left(t_{i}, W_{i}\right)^{\omega} \subseteq \bigcup D$. Thus, $\left\langle s, W_{m}\right\rangle \in D^{\prime}$, and because $\left\langle s, W_{m}\right\rangle \leq\langle s, W\rangle, D^{\prime}$ is dense in $\mathbb{M}_{\mathscr{U}}^{b}$.

Let $X_{G}$ be $\mathbb{M}_{\mathscr{U}}^{b}$-generic and let $Y \in\left(X_{G}\right)^{\omega}$ be arbitrary. Since $D^{\prime}$ is dense, there is a condition $\langle s, Z\rangle \in D^{\prime}$ such that $s \preccurlyeq X_{G} \sqsubseteq Z$. Since $Y \in$ $\left(X_{G}\right)^{\omega}$, we have $t \preccurlyeq Y \sqsubseteq Z$ for some $t \sqsubseteq s$ with $\operatorname{dom}(t)=\operatorname{dom}(s)$, and because $(t, Z)^{\omega} \subseteq \bigcup D$, we get $Y \in \bigcup D$. Hence, $Y \in \bigcup D$ for each dense set $D \subseteq \mathbb{M}_{\mathscr{U}}^{b}$, which completes the proof.

Appendix. In this section we gather some results concerning the dual form of some cardinal characteristics of the continuum. For the definition of the classical cardinal characteristics, as well as for relations between them, we refer the reader to [Va90].

First we consider the shattering cardinal $\mathfrak{h}$. This cardinal was introduced in [BPS80] as the minimal height of a tree $\pi$-base of $\beta \mathbb{N} \backslash \mathbb{N}$. Later it was shown by Szymon Plewik [Pl86] that $\mathfrak{h}=\operatorname{add}\left(r^{0}\right)=\operatorname{cov}\left(r^{0}\right)$, where $r^{0}$ denotes the ideal of Ramsey-null sets. It is easy to see that $\mathfrak{p} \leq \mathfrak{h}$, and therefore, $\operatorname{MA}(\sigma$-centered $)$ implies $\mathfrak{h}=\mathfrak{c}$.

The dual forms of the classical cardinal characteristics were introduced and investigated in [CW00] and further investigated in [Ha98 2 . Concerning the dual-shattering cardinal $\mathfrak{H}$, one easily gets $\aleph_{1} \leq \mathfrak{H} \leq \mathfrak{h}$, and in [Ha98 it is shown that $\mathfrak{H}>\aleph_{1}$ is consistent with ZFC and $\mathfrak{H}=\operatorname{add}\left(R^{0}\right)=\operatorname{cov}\left(R^{0}\right)$, where $R^{0}$ denotes the ideal of dual Ramsey-null sets. After all these symmetries, one would not expect the following: $\mathrm{MA}+(\mathfrak{c}>\mathfrak{H})$ is consistent with ZFC. This was proved by Jörg Brendle in [Br00] and implies that $\mathfrak{H}<\mathfrak{p}$ is consistent with ZFC. 
Concerning the reaping number $\mathfrak{r}$ and the dual-reaping number $\mathfrak{R}$, the situation is different. It is shown in $\left[\mathrm{Ha}_{9} 8_{1}\right]$ that $\mathfrak{p} \leq \mathfrak{R} \leq \min \{\mathfrak{r}, \mathfrak{i}\}$, and thus MA $(\sigma$-centered) implies $\mathfrak{R}=\mathfrak{c}$. Further, it is easy to show that $\mathfrak{R} \leq \mathfrak{U}$, where $\mathfrak{U}$ denotes the partition-ultrafilter base number, i.e., the dual form of $\mathfrak{u}$, and consequently, MA $(\sigma$-centered $)$ implies $\mathfrak{U}=\mathfrak{c}$.

For a Ramsey ultrafilter $\mathcal{U}$, Brendle [Br95] introduced the ideal $r_{\mathcal{U}}^{0}$ of Ramsey-null sets with respect to the ultrafilter $\mathcal{U}$. He showed for example that hom $\leq \operatorname{non}\left(r_{\mathcal{U}}^{0}\right)$, where $\mathfrak{h o m}$ is the homogeneity number investigated by Blass in [Bl93, Section 6]. There, Blass also investigated the so-called partition number $\mathfrak{p a r}$ and showed that $\mathfrak{p a r}=\min \{\mathfrak{b}, \mathfrak{s}\}$. Now, replacing the Ramsey ultrafilter $\mathcal{U}$ by a Ramseyan ultrafilter $\mathscr{U}$, one obtains the ideal $R_{\mathscr{U}}^{0}$ of dual Ramsey-null sets with respect to $\mathscr{U}$ as the dualization of the ideal $r_{\mathcal{U}}^{0}$, and replacing the colorings of $[\omega]^{2}$-involved in the definition of $\mathfrak{h o m}$ and $\mathfrak{p a r}$ - by colorings of $(\omega)^{2 *}$, one obtains the cardinal characteristics $\mathfrak{H o m}$ and $\mathfrak{P a r}$ and could begin to investigate them. But this is left to the reader.

\section{References}

[BPS80] B. Balcar, J. Pelant and P. Simon, The space of ultrafilters on $N$ covered by nowhere dense sets, Fund. Math. 110 (1980), 11-24.

[BJ95] T. Bartoszyński and H. Judah, Set Theory: On the Structure of the Real Line, A. K. Peters, Wellesley, 1995.

[Bl73] A. Blass, The Rudin-Keisler ordering of p-points, Trans. Amer. Math. Soc. 179 (1973), 145-166.

$\left[\mathrm{Bl}_{1} 1_{1} \quad-\right.$, Some initial segments of the Rudin-Keisler ordering, J. Symbolic Logic 46 (1981), 147-157.

[Bl81 $]$-, A partition theorem for perfect sets, Proc. Amer. Math. Soc. 82 (1981), 271-277.

[B193] - Simple cardinal characteristics of the continuum, in: Set Theory of the Reals, H. Judah (ed.), Israel Math. Conf. Proc. 6, Bar-Ilan Univ., 1993, 63-90.

[Bo70] D. Booth, Ultrafilters on a countable set, Ann. Math. Logic 2 (1970), 1-24.

[Br95] J. Brendle, Strolling through paradise, Fund. Math. 148 (1995), 1-25.

[Br00] - Martin's axiom and the dual distributivity number, Math. Logic Quart. 46 (2000), 241-248.

[CS84] T. J. Carlson and S. G. Simpson, A dual form of Ramsey's Theorem, Adv. Math. 53 (1984), 265-290.

[CW00] J. Cichon, A. Krawczyk, B. Majcher-Iwanow and B. Węglorz, Dualization of the van Douwen diagram, J. Symbolic Logic 65 (2000), 959-968.

[CN74] W. W. Comfort and S. Negrepontis, The Theory of Ultrafilters, Grundlehren Math. Wiss. 211, Springer, Berlin, 1974.

[El74] E. Ellentuck, A new proof that analytic sets are Ramsey, J. Symbolic Logic 39 (1974), 163-165.

[Ha981] L. Halbeisen, Symmetries between two Ramsey properties, Arch. Math. Logic 37 (1998), 241-260.

[Ha98 $]$-, On shattering, splitting and reaping partitions, Math. Logic Quart. 44 (1998), 123-134. 
$\left[\mathrm{HL} \infty_{1}\right]$ L. Halbeisen and B. Löwe, Ultrafilter spaces on the semilattice of partitions, Topology Appl., to appear.

$\left[\mathrm{HL}_{2}\right]-$ - - Techniques for approaching the dual Ramsey property in the projective hierarchy, Pacific J. Math., to appear.

[HS98] N. Hindman and D. Strauss, Algebra in the Stone-Čech Compactification: Theory and Applications, de Gruyter, New York, 1998.

[Je78] T. Jech, Set Theory, Academic Press, London, 1978.

[Ku83] K. Kunen, Set Theory. An Introduction to Independence Proofs, North-Holland, Amsterdam, 1983.

[La89] C. Laflamme, Forcing with filters and complete combinatorics, Ann. Pure Appl. Logic 42 (1989), 125-163.

[La84] R. Laver, Products of infinitely many perfect trees, J. London Math. Soc. (2) 29 (1984), 385-396.

[Mt86] P. Matet, Partitions and filters, J. Symbolic Logic 51 (1986), 12-21.

[Ma77] A. R. D. Mathias, Happy families, Ann. Math. Logic 12 (1977), 59-111.

[vM90] J. van Mill, An introduction to $\beta \omega$, in: Handbook of Set-Theoretic Topology, K. Kunen and J. E. Vaughan (eds.), North-Holland, Amsterdam, 1990, 503-567.

[P186] S. Plewik, On completely Ramsey sets, Fund. Math. 127 (1986), 127-132.

[Ra29] F. P. Ramsey, On a problem of formal logic, Proc. London Math. Soc. (2) 30 (1929), 264-286.

[Sh98] S. Shelah, Proper and Improper Forcing, Springer, Berlin, 1998.

[To97] S. Todorčević, Topics in Topology, Lecture Notes in Math. 1652, Springer, Berlin, 1997.

[Va90] J. E. Vaughan, Small uncountable cardinals and topology, in: Open Problems in Topology, J. van Mill and G. Reed (eds.), North-Holland, Amsterdam, 1990, $195-218$.

Department of Pure Mathematics

Queen's University Belfast

Belfast BT7 1NN, Northern Ireland

E-mail: halbeis@qub.ac.uk

Received 6 April 2000;

in revised form 13 March 2001 